\title{
Low prevalence of islet autoimmunity in adult diabetes and low predictive value of islet autoantibodies in the general adult population of northern Italy
}

\author{
E. Bosi ${ }^{1}$, M.P. Garancini ${ }^{2}$, F. Poggiali ${ }^{1}$, E. Bonifacio ${ }^{1}$, G. Gallus ${ }^{2}$ \\ ${ }^{1}$ Department of Medicine, San Raffaele Scientific Institute, University of Milan, Italy \\ ${ }^{2}$ Epidemiology Unit, San Raffaele Scientific Institute, University of Milan, Italy
}

\begin{abstract}
Aims/hypothesis. To assess the prevalence of islet autoimmunity in adult-onset diabetes mellitus and the predictive value of islet autoantibodies in the general adult population of northern Italy.

Methods. A sample of 2076 people aged 40 years or more participating in the population-based Cremona Study and classified in 1990 as having diabetes mellitus, impaired and normal glucose tolerance according to WHO criteria after an oral glucose tolerance test, were tested for antibodies to glutamic acid decarboxylase and IA-2.

Results. Increased concentrations of glutamic acid decarboxylase antibodies were found in $4(2.8 \%)$ of 143 participants with known diabetes and none of 50 with previously unknown diabetes, $1(0.65 \%)$ of 153 with impaired and $18(1.0 \%)$ of 1718 with normal glucose tolerance. The increased prevalence of these antibodies in subjects with known diabetes was not statistically significant. Protein tyrosine phosphatase IA-2-an-
\end{abstract}

tibodies were found in only four subjects, two of whom also had glutamic acid decarboxylase antibodies, all with normal glucose tolerance. After 8 years of follow-up, none of 21 non-diabetic subjects with either glutamic acid decarboxylase or IA-2-antibodies had developed diabetes and only a slight deterioration from normal to impaired fasting glucose was observed in 3 of 15 subjects with previous normal glucose tolerance.

Conclusion/interpretation. This study has shown that in northern Italy the prevalence of adult autoimmune diabetes in the general adult population is $0.19 \%$ (95\% CI 0.05-0.5); that autoimmune diabetes represents only a minority of all cases of adult diabetes; and that islet autoantibodies are not a high-risk factor for diabetes development in adults with normal glucose tolerance over 8 years of follow-up. [Diabetologia (1999) 42: 840-844]

Keywords Type I diabetes, Type II diabetes, autoantibodies, epidemiology, prediction.
Adult-onset diabetes mellitus is one of the most common chronic diseases of modern times. In 1990 the

Received: 29 December 1998 and in revised form: 9 March 1999

Corresponding author: E. Bosi, MD, Department of Medicine, Istituto Scientifico San Raffaele, Via Olgettina, 60, I-20132 Milan, Italy

Abbreviations: IFG, Impaired fasting glucose; WHO, World Health Organization; ADA, American Diabetes Association; GADA, glutamic acid decarboxylase antibodies; IA-2A, protein tyrosine phosphatase IA-2 antibodies; IAA, insulin autoantibodies; IA, insulin antibodies.
Cremona Study evaluated the prevalence of diabetes mellitus, based on the oral glucose tolerance test (OGTT) in accordance with the World Health Organization (WHO) criteria, at $9.4 \%$ in a sample representative of the general adult population of northern Italy [1]. Type II (non-insulin-dependent) diabetes mellitus is the predominant form of adult-onset diabetes mellitus. A proportion of adult patients with diabetes diagnosed as Type II develop insulin-requiring diabetes and many of these can be identified by the presence of circulating islet autoantibodies [2-4]. This form of diabetes is also known as latent autoimmune diabetes in adults (LADA) and, according to the new classification criteria, is now considered a 
form of Type I (insulin-dependent) diabetes mellitus or autoimmune diabetes.

Although it is thought that autoimmune diabetes in the adult is not infrequent, its prevalence in the general adult population and the proportion of patients classified as having Type II diabetes who are undiagnosed cases of Type I diabetes is still largely not known. To determine the prevalence of autoimmune diabetes and the predictive value of islet autoantibodies in a non-selected adult population with or without diabetes, we tested the 1990 cohort plasma samples of the Cremona Study for antibodies to glutamic acid decarboxylase (GADA) and protein tyrosine phosphatase IA-2 (IA-2A) and examined diabetes outcome in subjects with antibody markers after 8 years of follow-up.

\section{Materials and methods}

The Cremona Study. In 1990 the population-based "Cremona Study" was carried out in the Health District of Cremona, Lombardy region, northern Italy. The principal objective of this study was to estimate the prevalence of diabetes mellitus and impaired glucose tolerance (IGT) in a population sample representative of the general population of northern Italy. The protocol and methods of the study have been reported previously [1], with the exception of participants aged 40 to 44 years who were not included in that analysis. Briefly, 3599 subjects aged 40 years or more were randomly selected from 45638 adult inhabitants of three representative municipalities of the Cremona Health Disctrict, and were asked to participate in the study. Of these, 2096 subjects ( $58 \%$ ascertainment) accepted and were interviewed and visited by a physician. Fasting blood samples from the subjects with known diabetes and samples at fast and $2 \mathrm{~h}$ after a 75-g OGTT from all the others were obtained and subjects classified according to the WHO criteria. None of the subjects had Type I diabetes diagnosed before the age of 30 years. Of the 2096 subjects investigated, 2076 were available for antibody measurement. Of these, $143(6.9 \%)$ had known diabetes, another $50(2.4 \%)$ had diabetes diagnosed after OGTT (plasma glucose [means \pm SD]: basal 7.3 \pm 2.5 , 2-h $14.0 \pm 3.9 \mathrm{mmol} / \mathrm{l})$ the overall total being $193(9.3 \%)$ with diabetes, $153(7.4 \%)$ had IGT (plasma glucose: basal $5.5 \pm 0.7,2$-h $9.0 \pm 0.9 \mathrm{mmol} / \mathrm{l}$ ) and $1718(82.7 \%)$ had a normal glucose tolerance (plasma glucose: basal $5.0 \pm 0.5,2$-h $5.0 \pm 1.2 \mathrm{mmol} / \mathrm{l})$. A further 12 $(0.6 \%)$ subjects were unclassified. Measurement of GADA and IA-2A was undertaken in all subjects and those with raised concentrations of either GADA or IA-2A were contacted in September 1998 to undergo a brief interview and provide a fasting blood sample.

Autoantibody measurement. Plasma samples of all participants were screened for both GADA and IA-2A using a combined radiobinding assay [5]. All samples with concentrations above the 95th centile and all the follow-up samples were re-tested separately for GADA and IA-2A. Results were converted into arbitrary units $/ \mathrm{ml}$, by extrapolation from a standard curve. Thresholds for positivity from previous studies calculated using 178 control subjects (median age 12 years, range $1-40$ ) corresponded to 3 units/ml for GADA and 1 unit for IA-2A. The following results were obtained from these GADA and IA$2 \mathrm{~A}$ assays at the Combined Islet Autoantibody Workshop:
GADA, sensitivity $88 \%$, specificity $98 \%$, reproducibility $100 \%$; IA-2A, sensitivity $70 \%$, specificity $99 \%$, reproducibility $100 \%$. Insulin antibodies, either induced by exogenous insulin therapy (IA) or as autoantibodies (IAA), were measured in samples with raised GADA or IA-2A and in all follow-up samples using a competitive protein A-sepharose/protein G-sepharose insulin radiobinding and immunoprecipitation microassay [6]. The threshold for positivity, calculated using 97 control subjects (median age 11 years, range 2-59) corresponded to 5 units $/ \mathrm{ml}$.

Statistical analysis. Frequency differences were tested using Fisher's exact test. 95 \% Confidence Intervals (CI) we calculated using the exact method.

\section{Results}

Prevalence of islet autoantibodies. The 99th centile of GADA in the 1718 subjects with normal glucose tolerance was 3 units $/ \mathrm{ml}$, which corresponded to the threshold previously identified in subjects aged less than 40 years. Consequently, 18 (1\% prevalence, $95 \%$ CI $0.6-1.7$ ) of these subjects were considered to have increased GADA concentrations (Fig. 1). Of the 143 subjects with known diabetes 4 (2.8\% prevalence, 95\% CI 0.9-7.5) had increased GADA concentrations and 2 of these had been treated with insulin since 1973 and 1983 (Table 1). None of the 50 subjects with diabetes diagnosed after OGTT and 1 (0.65\% prevalence, $95 \%$ CI $0-4.0)$ of the 153 subjects with IGT had increased GADA concentrations (Fig.1). Overall, the prevalence of GADA-positive diabetes was $2.1 \%$ (95\% CI $0.7-5.6)$ of all participants with diabetes aged 40 years or more and $0.19 \%$ (95\% CI 0.05-0.5) of the general adult population of the same age. The increased GADA prevalence in subjects with known diabetes was not statistically significant (known diabetes vs normal glucose tolerance $p=0.07$ ).

Protein tyrosine phosphatase IA-2A above 1 unit/ $\mathrm{ml}$ were found in only four subjects, two of whom also had GADA (Fig. 1). Concentrations of IA-2A in these four subjects were $6.8,2.1,1.9$ and 1.5 units/ $\mathrm{ml}$ and all of them had a normal glucose tolerance (Table 1).

Of those subjects with raised GADA or IA-2A in 1990, five had raised insulin antibodies: the two with the highest concentrations were those on insulin treatment, whereas IAA at lower concentrations were detected in three other subjects, one with diabetes and two with normal glucose tolerance.

Subjects with increased concentrations of either GADA or IA-2A did not differ from subjects with undetectable antibodies with respect to sex (male proportion 60 vs $44 \%$, respectively), age (means \pm SEM: $55.2 \pm 2.1$ vs $57.6 \pm 0.2$ years, respectively) and body mass index $\left(26.9 \pm 0.9\right.$ vs $26.5 \pm 0.1 \mathrm{~kg} / \mathrm{m}^{2}$, respectively). 


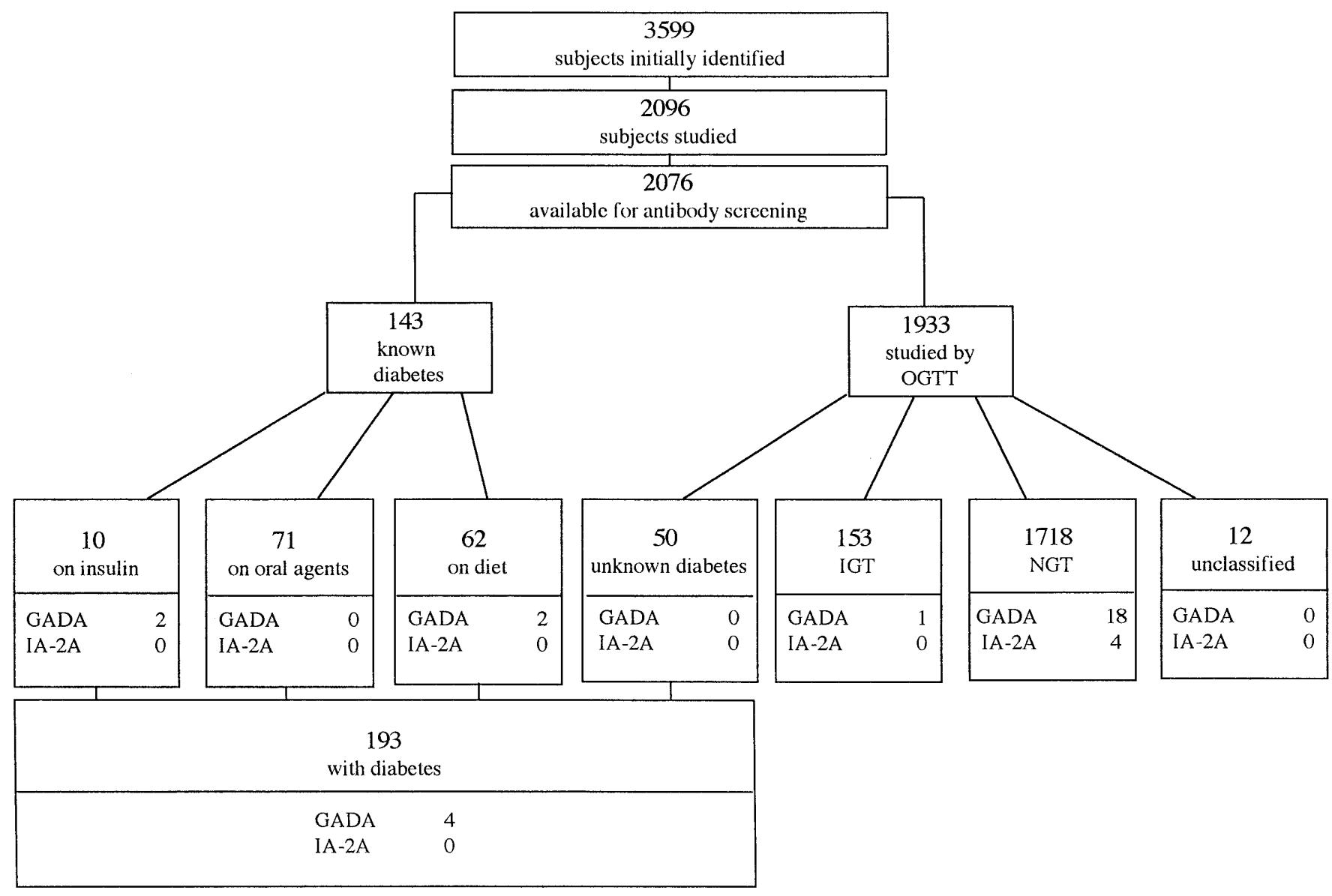

Fig. 1. Study profile and distribution of autoantibodies

8 year follow-up. Of the 25 subjects with raised islet autoantibodies in 1990, 21 were still alive in 1998. Information was obtained for 19 and a blood sample in fasting conditions from 16 (Table 1 ). Of these, 1 was already diabetic on insulin, and none of the other 15 subjects with normal glucose tolerance in 1990 developed diabetes. An impaired fasting glucose in 1998 was present in three of these subjects, one having GADA, IA-2A and IAA and the other two having GADA only. None of the others showed changes in their glucose metabolism regulation. Of the four subjects who died, two had diabetes, one of whom had been on insulin therapy, one had IGT and one had a normal glucose tolerance. In 1998, GADA remained raised in 11 of 15 subjects with GADA, IA-2A in none of 3 subjects and IAA in none of 2 subjects having antibodies in 1990. Subjects in whom antibodies were no longer detected had relatively low antibody titres in their 1990 sample (Table 1).

\section{Discussion}

This study shows a low prevalence of adult Type I diabetes in the general population of northern Italy. Only $2 \%$ of participants having known or unknown diabetes from the general adult population had raised GADA levels, resulting in a prevalence of adult autoimmune diabetes in the general population of $0.19 \%$. Note that, these data may slightly underestimate the overall prevalence of adult Type I diabetes since the current study did not test islet cell antibodies, which have been shown to identify additional patients with autoimmune diabetes, especially in the older classes of age [3]. Nonetheless, although low and with a large confidence interval, the prevalence of adult Type I diabetes is higher than that of the disease diagnosed before the age of 30 , which in the same area and in the same classes of age is $0.06 \%$, as can be estimated from a previous study [7].

These figures are close to those reported in a similar study in The Netherlands, the only other study reporting the prevalence of autoimmune diabetes in the general adult population [8]. Several other studies reported islet autoantibodies at much higher frequencies in patients with adult-onset diabetes [2-4], leading to the impression that autoimmunity accounts for a considerable proportion of patients diagnosed in older age. Reasons for such discrepancies include 
Table 1. Antibody concentrations and glucose metabolism category of the 25 subjects with either GADA or IA-2A

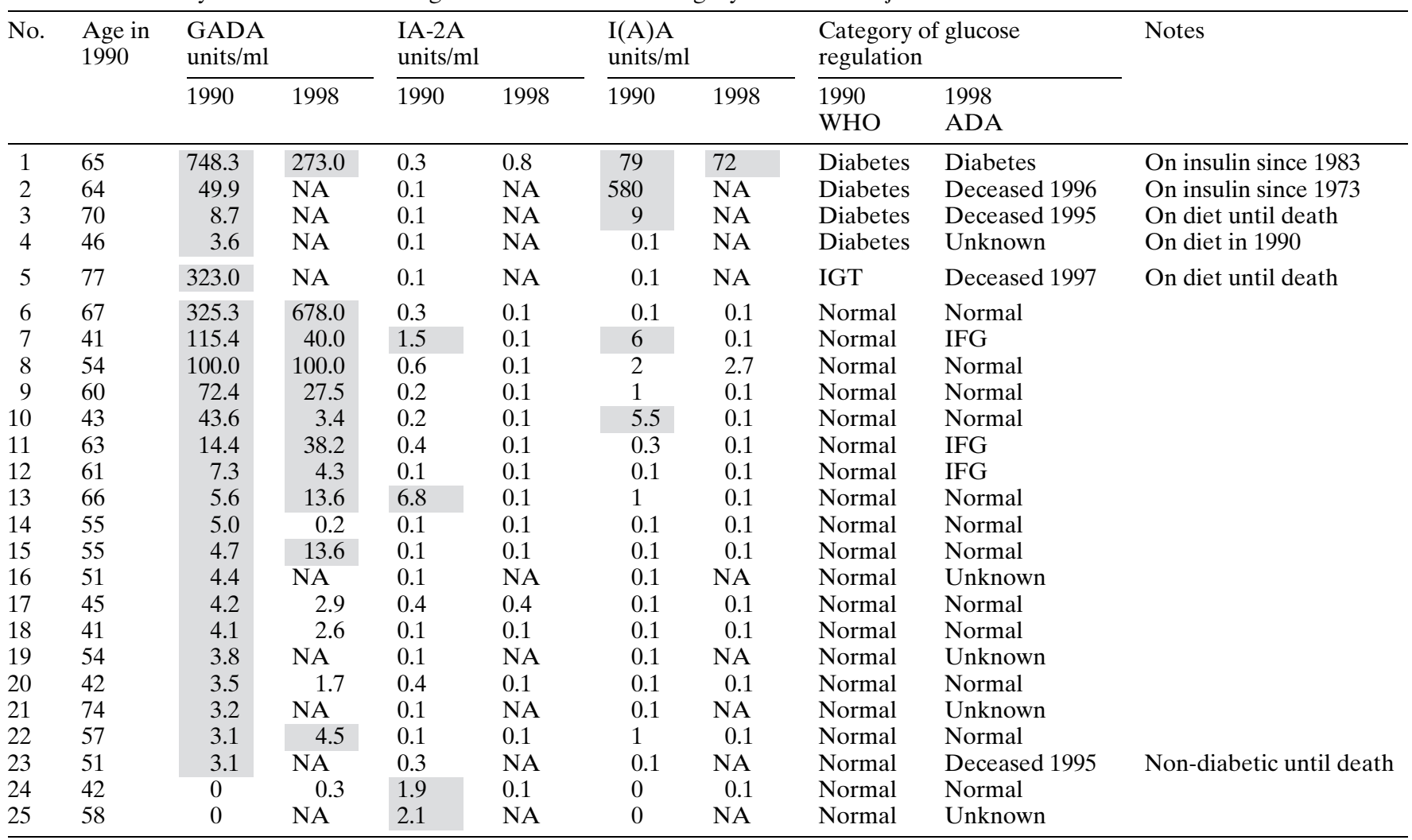

Antibodies values above the threshold for positivity are reported in bold and within shaded areas. I(A)A indicates IA or IAA. WHO indicates World Health Organization criteria and ADA American Diabetes Association criteria for classification of glucose metabolism regulation categories. NA: not available ethnic and regional differences in the incidence of Type I diabetes within Europe, where the Lombardy region shows one of the lowest incidence rates of childhood diabetes [9], and differences in patient selection, which in those studies was mainly based on hospital or clinic attendance, resulted in an enrichment of Type I diabetes.

A relevant finding of this study is the evidence of a low predictive value of GADA for the development of diabetes in adults with normal glucose tolerance. None of the 15 subjects with normal glucose tolerance and either GADA or IA-2A developed insulin dependence or even diabetes over a period of 8 years of follow-up. Only a marginal deterioration of fasting glucose concentrations was observed in three subjects with islet autoantibodies who progressed from normal to impaired fasting glucose. These data are similar to those found in relatives of Type I diabetic patients and in the general population of schoolchildren where the presence of GADA, or any other islet autoantibody when detected alone, is associated with a relatively low risk of developing Type I diabetes [10].

In conclusion, we have shown that adult autoimmune Type I diabetes represents only $2 \%$ of all adults with diabetes and affects around $0.2 \%$ of the general adult population of northern Italy. The prevalence of GADA is slightly increased in adult patients with diabetes compared with patients of the same age with normal glucose tolerance and IA-2A are extremely rare in adults. Although they are a diagnostic marker of adult Type I diabetes, GADA, even at high concentrations, do not seem to be highly predictive of future diabetes when they are found in adults with normal glucose tolerance.

Acknowledgements. The authors thank V. Bettamio for assistance in patient interviewing and blood sampling, C. Belloni for insulin antibody measurement and A. Venerando for assistance in GADA and IA-2A measurement.

\section{References}

1. Garancini MP, Calori G, Ruotolo G et al. (1995) Prevalence of NIDDM and impaired glucose tolerance in Italy: an OGTT-based population study. Diabetologia 38: 306-313

2. Hagopian WA, Karlsen AE, Gottsater A et al. (1993) Quantitative assay using recombinant human islet glutamic acid decarboxylase (GAD65) shows that 64K autoantibody positivity at onset predicts diabetes type. J Clin Invest 91: 368-374

3. Turner R, Stratton I, Horton V et al. (1997) UKPDS 25: autoantibodies to islet cell cytoplasm and glutamic acid decar- 
boxylase for prediction of insulin requirement in type 2 diabetes. Lancet 350: 1288-1293

4. Tuomi T, Carlsson AL, Li H et al. (1999). Clinical and genetic characteristics of type 2 diabetes with and without GAD antibodies. Diabetes 48: 150-157

5. Bonifacio E, Lampasona V, Genovese S, Ferrari M, Bosi E (1995) Identification of Protein Tyrosine Phosphatase-like IA2 (islet cell antigen 512) as the insulin-dependent diabetes-related $37 / 40 \mathrm{~K}$ autoantigen and a component of islet cell antibodies. J Immunol 155: 5419-5426

6. Naserke HE, Dozio N, Ziegler AG, Bonifacio E (1998) Comparison of a novel microassay for insulin autoantibodies with the conventional radiobinding assay. Diabetologia 41: 681-683
7. Garancini MP, Sergi A, Lazzari P, Gallus G (1995) Epidemiology of known diabetes in Lombardy, north Italy. Clinical characteristics and methodological aspects. Acta Diabetol 32: 268-272

8. Ruige JB, Batstra MR, Aanstoot HJ et al. (1997) Low prevalence of antibodies to GAD65 in a 50 to 74 year old general dutch population. Diabetes Care 20: 1108-1110

9. Green A, Gale EAM, Patterson CC, for the EURODIAB ACE Study Group (1992) Incidence of childhood-onset insulin-dependent diabetes mellitus: the EURODIAB ACE Study Group. Lancet 339: 905-909

10. Bingley PJ, Bonifacio E, Williams AJK, Genovese S, Bottazzo GF, Gale EAM (1997) Prediction of IDDM in the general population: strategies based on combinations of autoantibody markers. Diabetes 46: 1701-1710 